\title{
Control of Hypersonic Aerodynamic Forces with Surface Blowing
}

\author{
M. D. Matarrese, ${ }^{*}$ A. F. Messiter, $\dagger$ and T. C. Adamson Jr. \\ University of Michigan, Ann Arbor, Michigan 48109
}

\begin{abstract}
Pressure forces are derived for laminar flow past a thin wedge at high Mach number and high Reynolds number, with mass added at the surface according to a power-law velocity distribution strong enough that the boundary layer is blown away from the wall as a free shear layer. Self-similar solutions are obtained for the thin layer of blown gas adjacent to the surface, for the thin viscous shear layer, and for the outer inviscid-flow region between the shear layer and the shock wave. Pressures obtained in the strong- and weak-interaction regions are joined by a simple interpolation formula. Integrated pressure forces are shown for a range of Mach numbers and altitudes, for various wedge lengths and vertex angles, and for different injected gases. Equilibrium dissociation of oxygen in the shear layer is found to have only a small effect on the pressure forces.
\end{abstract}

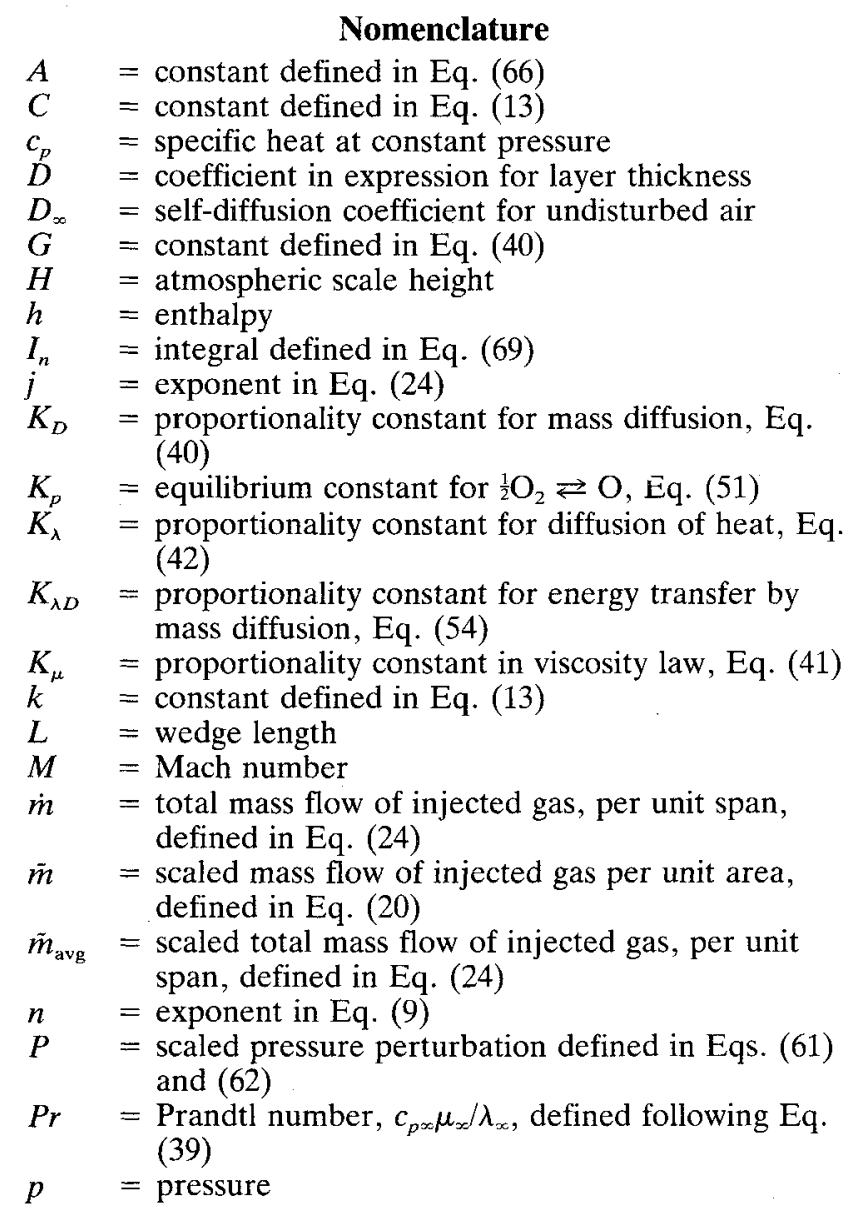

Presented as Paper 90-0602 at the AIAA 28th Aerospace Sciences Meeting, Reno, NV, Jan. 8-11, 1990; received May 30, 1990; revision received Dec. 10, 1990; accepted for publication Dec. 20, 1990. Copyright (c) 1989 by the authors. Published by the American Institute of Aeronautics and Astronautics, Inc., with permission.

*Graduate Student, Department of Aerospace Engineering. Student Member AIAA.

$\dagger$ Professor, Department of Aerospace Engineering. Associate Fellow AIAA.

$¥$ Professor, Department of Aerospace Engineering. Fellow AIAA
$Q \quad=$ scaled mass flow defined in Eqs. (61) and (62)

$q \quad=$ exponent in Eq. (43)

$R \quad=$ nondimensional gas constant for mixture, defined in Eq. (35)

$R e \quad=$ Reynolds number, $u_{\infty} L / \nu_{\infty}$

$r \quad=$ function of $x$ defined in Eq. (66)

Sc $=$ Schmidt number, $\mu_{\infty} /\left(\rho_{\infty} D_{\infty}\right)$, defined following Eq. (39)

$T \quad=$ temperature

$u, v=X$ and $Y$ components of velocity, respectively

$X, Y=$ coordinates along and normal to undisturbed flow, respectively

$X_{r} \quad=$ reference length defined in Eq. (1)

$x, y=$ nondimensional coordinates along and normal to undisturbed flow, respectively

$Y_{b} \quad=$ mass fraction of blown gas

$Y_{O}=$ mass fraction of atomic oxygen

$z \quad=$ altitude

$\alpha \quad=$ wedge vertex half-angle

$\beta=$ shock-wave inclination angle

$\gamma \quad=$ ratio of specific heats

$\Delta \quad=$ layer thickness

$\Delta F \quad=$ increase in normal force on wedge, above inviscid-flow value

$\varepsilon \quad=$ fraction of molecules that dissociate into oxygen atoms, Eq. (50)

$\zeta \quad=$ similarity variable defined in Eq. (43)

$\zeta_{1} \quad=$ lower limit for $\zeta$

$\lambda=$ thermal conductivity

$\mu \quad=$ viscosity coefficient

$\nu \quad=$ kinematic viscosity

$\rho \quad=$ density

$\sigma_{b} \quad=$ ratio of molecular weight of blown gas to that of air

$\phi \quad=$ defined in Eq. (63)

$\chi \quad=$ defined in Eq. (64)

$\underline{\Psi}=$ nondimensional stream function

$\bar{\Psi}_{l}, \bar{\Psi}_{u}=$ lower and upper limits of $\bar{\Psi}$ in Eq. (55)

$\omega \quad=$ exponent in viscosity law

Subscripts

$$
\begin{array}{ll}
a & =\text { air } \\
b & =\text { blown gas } \\
s & =\text { value at shock wave } \\
w & =\text { value at wall } \\
0 & =\text { (scaled) quantity in inviscid wedge flow } \\
1 & =\text { coefficient in perturbation quantity } \\
\infty & =\text { undisturbed-flow value }
\end{array}
$$


Superscripts

(s) = strong-interaction region

(w) = weak-interaction region

$=$ nondimensional quantity in outer inviscid-flow region

$\sim \quad=$ nondimensional quantity in blown-gas layer
$-\quad=$ nondimensional quantity in viscous shear layer

\section{Introduction}

C ONTROL of flight vehicles at hypersonic speeds has usually been achieved by means of moving control surfaces and/or by changing the magnitude and direction of the thrust vector. As a possible alternative method of control, it might be of interest to consider the use of boundary-layer blowing to introduce desired modifications in pressure distributions over the surface and thus to provide control forces on a specified part of a vehicle. Different injection patterns would be possible, including distributed or strip blowing. In the present study a simple blowing distribution is considered for the simplest possible geometry, a thin wedge in two-dimensional hypersonic flow. If the mass rate of flow of injected gas is sufficiently large, the boundary layer is completely blown off from the surface; this is the case considered here. The solutions can be useful in several contexts. First, they provide a measure of the magnitudes of forces to be expected for a given blowing rate at given flight conditions; second, they can be used to check computer codes developed to handle more complex flow problems ${ }^{1}$; finally, they may have some application in the related problems of cooling hypersonic vehicles by boundary-layer blowing, and of the introduction of fuel into the combustion region of a scramjet.

Hypersonic flows of a perfect gas past simple shapes have been studied extensively using inviscid-flow theory, and viscous-interaction effects are also well understood. For a thin wedge, the effects of small perturbations in surface shape require solution of linearized equations. ${ }^{2}$ If the perturbations are caused by the displacement thickness of a laminar boundary layer, the equations for a constant-pressure boundary layer at high Mach number allow a complete description of the flow at points far enough from the leading edge. In a region closer to the vertex where the boundary-layer thickness is large compared to the wedge thickness (but streamline slopes are still small), a self-similar solution is available, ${ }^{3,4}$ based on the hypersonic small-disturbance equations ${ }^{5}$ for the shock layer and boundary-layer equations for the viscous layer. The interactions of the boundary layer with the external inviscid flow in these two regions are, respectively, "weak" and "strong" interactions.

Surface injection of a gas in the strong-interaction region, for a suitable power-law velocity variation, still allows a selfsimilar formulation but with modified boundary conditions. $\mathrm{Li}$ and Gross ${ }^{6}$ obtained numerical results for strong interaction using boundary-layer solutions with a nonzero normal velocity component at the surface, over a range of prescribed surface temperatures. If the blowing is strong enough, however, the boundary layer is blown away from the surface. Kubota and Fernande $z^{\text {? }}$ considered a compressible free shear layer, with prescribed power-law pressure gradient, and an additional thin layer of inviscid flow adjacent to the wall. For still stronger blowing velocities, the layer of blown gas is much thicker than the free shear layer and the shear layer can be regarded as a surface of discontinuity. Cole and Aroesty ${ }^{8}$ described the blown gas by "inviscid boundary-layer equations" and the shock layer again by hypersonic small-disturbance equations. A useful summary of this and earlier related work was given by Smith and Stewartson. ${ }^{9}$

When the blowing velocity has suitable strength, as well as an appropriate power-law variation, in the strong-interaction region, the displacement effects of the blown gas and of the shear layer are of the same order of magnitude. ${ }^{10}$ Solutions in the strong-interaction limit can then be obtained inde- pendently for the thin layer of blown gas, the viscous free shear layer, and the inviscid shock layer, with certain constants to be found by proper asymptotic matching. The shock layer is described by the hypersonic small-disturbance equations, ${ }^{5}$ the viscous layer by the usual boundary-layer equations, and the blown gas by inviscid boundary-layer equations. ${ }^{8}$ The derivations of Ref. 10 were given primarily for a flat plate, with an application to a thin wedge mentioned relatively briefly. Since the temperature in the shear layer is high, the scaled temperature in the shear layer should approach zero both above and below. Numerical solution of the shear-layer equations was carried out by a continuation method in which the mass flow entrained in the lower part of the shear layer is varied until the proper asymptotic behavior is observed. ${ }^{11}$ The weak-interaction region can also be described by self-similar solutions; an approximate interpolation formula was proposed in Ref. 10 for joining the surface pressures found from the strong- and weak-interaction solutions.

The present study is concerned with pressure forces acting on a thin wedge in the presence of mass injection at the surface having a power-law dependence on distance from the vertex. The derivations of Ref. 10 are extended in several ways: to allow constant surface temperature rather than constant surface density, as assumed in Ref. 10 to achieve some minor simplifications; to allow arbitrary values of the hypersonic similarity parameter instead of requiring that the normal Mach number at the shock wave be large; to allow an injected gas different from air; to allow for variable specific heats and oxygen dissociation in the viscous shear layer, and to provide a variety of numerical results for pressure forces caused by blowing.

The main objective is to relate the pressure distribution at the wedge surface to the prescribed surface conditions, expressed by the specified distribution of injected mass and either a constant wall density or a constant wall temperature. Solutions for the inviscid outer flow provide the relation between the surface pressure and the effective body shape; solutions for the inviscid layer of blown gas give an expression for the thickness of this layer in terms of the surface pressure and the Mach number at the wall, here expressed in terms of the surface mass flux and the temperature or density at the wall; the solution for the viscous shear layer provides a relation between the shear-layer thickness and the surface pressure. Combining these results with the help of first-order matching conditions then yields the desired results.

The following section summarizes the analysis, with emphasis on the new aspects. Numerical results for integrated forces are then shown and discussed for a range of Mach numbers and altitudes, for various wedge lengths and vertex angles, and for different injected gases at different values of surface temperature.

\section{Analysis}

A thin wedge having length $L$ and small vertex half-angle $\alpha \ll 1$ is placed at zero incidence in a uniform hypersonic flow with Mach number $M_{\infty} \gg 1$. A power-law viscositytemperature relation $\mu / \mu_{\infty}=\left(T / T_{\infty}\right)^{\omega}$ is assumed for air; if a different gas is injected at the wedge surface, $\mu$ also depends on the mass fraction $Y_{b}$ of the blown gas.

In the absence of surface blowing, an effective body shape is found by adding the boundary-layer displacement thickness to the location of the wedge surface $Y \sim \alpha X$. At points sufficiently far from the vertex (when $L$ is large enough), the boundary-layer thickness $\mathscr{O}\left\{M_{x}^{\omega} \alpha^{-1}\left(\nu_{x} X / u_{x}\right)^{1 / 2}\right\}$, where $\nu_{x}=$ $\mu_{\infty} / \rho_{\infty}$, is small in comparison with one-half the wedge thickness $\alpha X$, and the pressure is nearly the same as for inviscid flow. At points closer to the leading edge, however, this is no longer true. A reference length $X_{r}$ is defined by equating the two thicknesses, so that for $X \gg X$, the boundary layer has only a small effect on the external flow, and the interaction is "weak," whereas for $X \ll X$, the boundary-layer displace- 
ment effect is dominant, and the interaction is "strong." 3.4 Nondimensional coordinates are then defined by

$$
\begin{gathered}
x=X / X_{r}, \quad y=(Y-\alpha X) /\left(\alpha X_{r}\right) \\
X_{r}=M_{\infty}^{2 \omega} \alpha^{-4} \nu_{\infty} / u_{\infty}
\end{gathered}
$$

where $\alpha \ll 1 ; y=0$ at the wedge surface (in the small-angle approximation); and at the shock wave $y=\mathcal{O}(1)$ when $x=$ $\mathcal{O}(1)$. The ratio $L / X_{r}$ is proportional to the Reynolds number $R e=u_{\infty} L / \nu_{\infty}$ based on wedge length and freestream quantities. For constant Mach number and an isothermal atmosphere, the length $X_{r}$ increases exponentially with altitude; a weaker dependence on temperature can be introduced by assuming a standard atmosphere. At low altitudes $X_{r}$ might be small in comparison with $L$; at sufficiently high altitudes $X_{r} \gg L$. The stream function, nondimensional with $\alpha \rho_{\infty} u_{\infty} X_{r}$, is defined by

$$
\rho_{\infty} \mu_{\infty} \Psi_{y}=\rho u
$$

with $\Psi=0$ along the streamline from the vertex of the wedge.

If mass is added to the flow by surface blowing, here taken as directed normal to the surface, the effective body thickness is increased further, and for sufficiently strong injection the boundary layer is blown away from the surface as a free shear layer. The equivalent body now includes the effects of both blowing and viscous diffusion. The sketches in Fig. 1 suggest the qualitative features of the flow in the strong- and weakinteraction regions, for weak blowing when the boundary layer is still attached and for strong blowing; in each case, the speckled region is the viscous layer. For the "strong" blowing considered here, three thin flow layers can be distinguished: a nearly inviscid thin layer of blown gas; a viscous free shear layer where mixing (assumed laminar) of the blown gas and freestream gas takes place; and an outer inviscid flow between the shear layer and the shock wave. Qualitative sketches of the mass-fraction, velocity, temperature, and pressure profiles for $x=\mathscr{O}(1)$ are shown in Fig. 2 for the entire region of disturbed flow between the wedge surface and the shock wave. Strong and weak interactions occur for $x<1$ and $x \gg 1$, respectively, and in general the flow variables will have different orders of magnitude in the different flow regions. The injectant temperature at the surface will be taken to have the same order of magnitude (other choices of course are possible) as the temperature in the undisturbed air, whereas the temperature in the viscous layer is larger by a factor $O\left(M_{\infty}^{2}\right)$. The mass flow in the blown layer is small because the flow velocities are small there, and in the viscous layer the mass flow is still smaller because the density is low; the first approximation to the pressure is a function only of $x$ in both regions. The amount of injected mass is chosen such that the blownlayer thickness is of the same order of magnitude as the wedge thickness when $x=\mathcal{O}(1)$. The thickness of the viscous layer

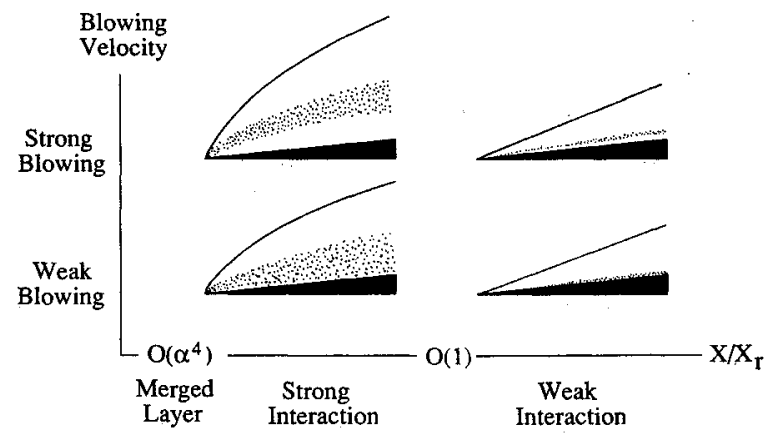

Fig. 1 Qualitative sketch of wedge flowfield with weak or strong blowing and weak or strong interaction. (Speckled regions are viscous layers.) is likewise of this order when $x=O(1)$; if, however, the mass flow is increased further, the viscous-layer thickness can be neglected.

\section{Inviscid Outer Flow}

The location $y=\Delta(x)$ of the equivalent body surface, flow variables in the region between this surface and the shock wave, and the stream function can be written as

$$
\begin{aligned}
& \Delta(x)=\bar{\Delta}(x)+\bar{\Delta}(x), \quad p / p_{\infty}=M_{\infty}^{2} \alpha^{2} \hat{p}(x, y)+\cdots \\
& \rho / \rho_{\infty}=\hat{\rho}(x, y)+\cdots \\
& u / u_{\infty}=1+\cdots, \quad \Psi=\hat{\Psi}
\end{aligned}
$$

where $\bar{\Delta}(x)$ and $\bar{\Delta}(x)$ describe the contributions of the blown gas and the shear layer, respectively. The pressure at the displacement surface $y=\Delta(x)$ can be found in terms of $\Delta(x)$ in both the strong- and weak-interaction limits, for suitably specified mass injection rates.

For $x \ll 1$, a self-similar solution describes strong viscous interaction. The surface of the equivalent body and the pressure at this surface have the form

$\Delta(x)=\Delta_{1}^{(s)} x^{3 / 4}+\cdots, \quad \hat{p}^{(s)}(x, \Delta(x))=p_{1}^{(s)} x^{-1 / 2}+\cdots$

where $p_{1}^{(s)}$ is a constant to be determined; the required form for surface blowing is shown below. The flow in the inviscid shock layer is described by the self-similar form of the hypersonic small-disturbance equations, with appropriate exponents. Numerical solution of these equations, for $\gamma_{x}=1.4$, gives a relation ${ }^{10}$ between the coefficients in Eqs. (4):

$$
\Delta_{1}^{(s)}=\hat{D}^{(s)}\left(p_{1}^{(s)}\right)^{1 / 2}, \quad \hat{D}^{(s)}=0.945
$$

The numerical value agrees with that of Ref. 6. The representation given by Eqs. (4) requires that streamline slopes be small and that the pressure be small in comparison with the dynamic pressure in the undisturbed flow. Thus, the solution fails for very small $x$, in the "merged-layer regime" for $x=$ $\mathcal{O}\left(\alpha^{4}\right)$, or $X=\mathcal{O}\left(M_{\infty}^{2 \omega} \nu_{x} / u_{\infty}\right)$; boundary-layer approximations fail here as well, as noted later.
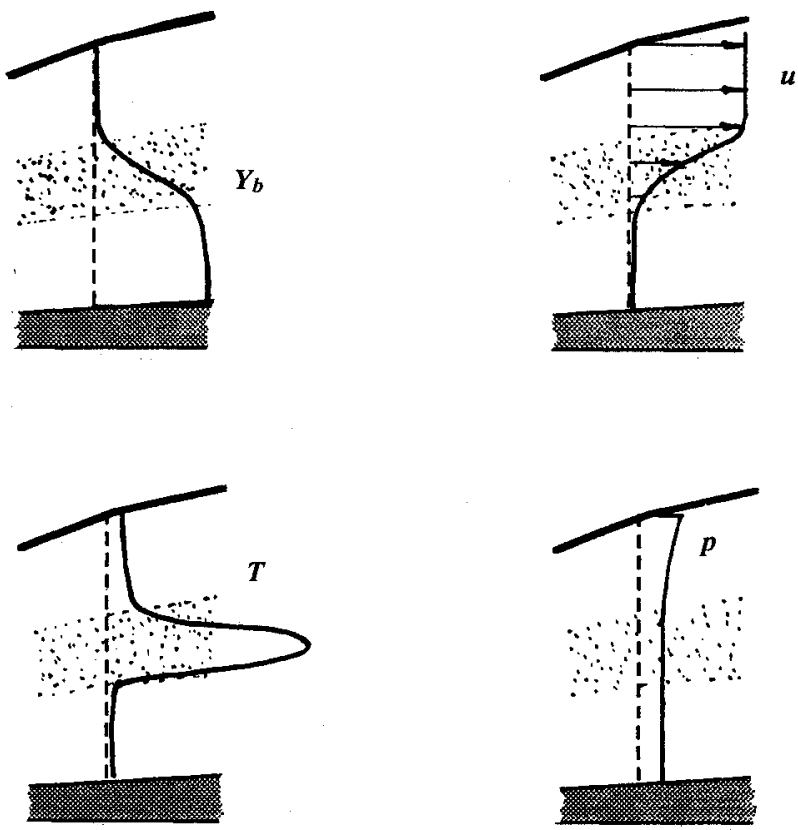

Fig. 2 Qualitative profiles of blown-gas mass fraction $Y_{b}$, velocity component $u$, temperature $T$, and pressure $p$. 
If $X_{r}<<L$, the strong interaction is confined to a small fraction of the wedge length. Further downstream, for the portion of the wedge where $x>>1$, the boundary layer causes only small disturbances to the outer inviscid flow. In the hypersonic small-disturbance approximation, the shock-wave jump conditions can be combined in the form

$$
\frac{v_{s}}{\beta u_{\infty}}=1-\frac{\rho_{\infty}}{\rho_{s}}=\frac{p_{s}-p_{\infty}}{\gamma_{\infty} M_{\infty}^{2} \beta^{2} p_{\infty}}=\frac{2}{\gamma_{\infty}+1}\left(1-\frac{1}{M_{\infty}^{2} \beta^{2}}\right)
$$

The flow variables for $x>>1$ have the form:

$$
\begin{gathered}
p / p_{\infty}=M_{\infty}^{2} \alpha^{2}\left\{p_{0}+\hat{p}_{1}^{(w)}(x, y)+\cdots\right\} \\
\rho / \rho_{\infty}=\rho_{0}+\cdots, \quad v / u_{\infty}=\alpha\left[1+\hat{v}_{1}^{(w)}(x, y)+\cdots\right\} \\
\beta=\alpha \beta_{0}+\cdots, \quad M=\alpha^{-1} M_{0}+\cdots
\end{gathered}
$$

The values for turning through a small angle $\alpha$ are given by

$$
\begin{gathered}
\beta_{0}=\frac{\gamma_{x}+1}{4}+\left\{\left(\frac{\gamma_{x}+1}{4}\right)^{2}+\frac{1}{M_{\infty}^{2} \alpha^{2}}\right\}^{1 / 2} . \\
\rho_{0}=\frac{\beta_{0}}{\beta_{0}-i}, \quad p_{0}=\gamma_{\infty} \beta_{0}+\frac{1}{M_{\infty}^{2} \alpha^{2}}, \quad M_{0}^{2}=\frac{\rho_{0}}{p_{0}}
\end{gathered}
$$

The product $M_{x} \alpha$ is the usual similarity parameter of hypersonic small-disturbance theory.

For the weak-interaction region $x>1$, the surface $y=$ $\Delta(x)$ of the equivalent body lies very close to the wedge surface. The body shape and the linearized boundary condition are

$$
\Delta(x)=\Delta_{1}^{(w)} x^{n}+\cdots, \quad \hat{v}_{1}^{(w)}(x, 0)=n \Delta_{1}^{(w)} x^{n-1}+\cdots
$$

where the value of $n<1$ depends on the choice of prescribed conditions at the surface. The shock-wave jump conditions given by Eqs. (6) provide a relation between the perturbations $\hat{p}_{1}^{(w)}$ and $\hat{v}_{1}^{(w)}$ at the linearized shock position $Y=\alpha \beta_{0} X$ or $y$ $=\left(\beta_{0}-1\right) x$ :

$$
\begin{aligned}
& \gamma_{\infty}^{-1} \hat{p}_{1}^{(w)}\left(x,\left(\beta_{0}-1\right) x\right) \\
& =\frac{\beta_{0}^{2}}{\beta_{0}-\left(\gamma_{\infty}+1\right) / 4} \hat{v}_{1}^{(w)}\left(x,\left(\beta_{0}-1\right) x\right)
\end{aligned}
$$

Each of the functions $\hat{p}_{1}^{(w)}$ and $\hat{v}_{1}^{(w)}$ must satisfy a wave equation in the $x, y$ coordinates, and the boundary conditions require that each have a self-similar form. The solutions satisfying these conditions are

$$
\begin{aligned}
\gamma_{x}^{-1} \hat{p}_{1}^{(w)} & =c_{1}\left(x+M_{0} y\right)^{n-1}+c_{2}\left(x-M_{0} y\right)^{n-1} \\
-\left(\rho_{0} / M_{0}\right) \hat{v}_{1}^{(w)} & =c_{1}\left(x+M_{0} y\right)^{n-1}-c_{2}\left(x-M_{0} y\right)^{n-1}
\end{aligned}
$$

where the constants are defined by

$$
\begin{gathered}
\frac{c_{1}}{C}=\frac{c_{2}}{k^{1-n}}=\frac{\rho_{0} n \Delta_{1}^{(w)}}{\left(k^{1-n}-C\right) M_{0}}, \quad k=\frac{1-M_{0}\left(\beta_{0}-1\right)}{1+M_{0}\left(\beta_{0}-1\right)} \\
C=\frac{M_{0} \beta_{0}\left(\beta_{0}-1\right)-\left[\beta_{0}-\left(\gamma_{\infty}+1\right) / 4\right]}{M_{0} \beta_{0}\left(\beta_{0}-1\right)+\left[\beta_{0}-\left(\gamma_{\infty}+1\right) / 4\right]}
\end{gathered}
$$

The pressure at the surface of the equivalent body and the shape of the body are related by

$$
\begin{gathered}
\Delta_{1}^{(w)}=\hat{D}^{(w)} p_{1}^{(w)}=x^{1-n} \hat{D}^{(w)} \hat{p}_{1}^{(w)}(x, 0) \\
\hat{D}^{(w)}=\frac{M_{0}\left(k^{1-n}-C\right)}{\gamma_{\infty} n \rho_{0}\left(k^{1-n}+C\right)}
\end{gathered}
$$

Thus, $\hat{D}^{(w)}$ depends on $\gamma_{x}, n$, and $M_{x} \alpha$. The results are equivalent to those of Ref. 2 .

\section{Inviscid Blown-Gas Layer}

At the wedge surface, gas is injected in a direction normal to the wall at a low Mach number $M_{w}=O(\alpha)$. For the blowing rates considered here, most of the injected gas occupies a thin layer adjacent to the surface; the flow in this blown-gas layer is nearly inviscid, and only a small fraction of the injected mass is entrained in the mixing layer. In the layer of blown gas, the scalings for the flow variables are essentially the same as suggested by Cole and Aroesty, ${ }^{8}$ and are found from the boundary and matching conditions, thermodynamic relations, and the conservation laws to have the form

$$
\begin{gathered}
p / p_{\infty}=M_{\infty}^{2} \alpha^{2} \tilde{p}(x)+\cdots, \quad \rho / \rho_{\infty}=M_{\nsim}^{2} \alpha^{2} \sigma_{b} \tilde{\rho}(x, y)+\cdots \\
T / T_{\infty}=\tilde{T}(x, y)+\cdots \\
u / u_{\infty}=\frac{1}{M_{\infty}}\left(\frac{\gamma_{b}}{\sigma_{b} \gamma_{\infty}}\right)^{1 / 2} \tilde{u}(x, y)+\cdots \\
v / u_{\infty}=\frac{\alpha}{M_{\infty}}\left(\frac{\gamma_{b}}{\sigma_{b} \gamma_{\infty}}\right)^{1 / 2} \tilde{v}(x, y)+\cdots
\end{gathered}
$$

The pressure is a function of $x$ in the first approximation since the layer is thin and the gas velocity is low. The stream function $\Psi$ is then

$$
\Psi=M_{\infty} \alpha^{2}\left(\frac{\sigma_{b} \gamma_{b}}{\gamma_{\infty}}\right)^{1 / 2} \tilde{\Psi}
$$

The streamline, total-enthalpy, entropy, and state equations become

$$
\begin{gathered}
y_{\tilde{\Psi}}=\frac{1}{\tilde{\rho} \tilde{u}}, \quad \frac{1}{2} \tilde{u}^{2}+\frac{\tilde{T}}{\gamma_{b}-1}=\frac{\tilde{T}_{w}}{\gamma_{b}-1} \\
\tilde{\rho}=\left(\frac{\tilde{p}}{\tilde{p}_{w}}\right)^{1 / \gamma_{b}} \tilde{\rho}_{w}, \quad \tilde{p}=\tilde{\rho} \tilde{T}
\end{gathered}
$$

This is the set of equations studied by Cole and Aroesty. ${ }^{8}$ The wall values $\tilde{\rho}_{w}, \tilde{v}_{w}, T_{w}$, and $\tilde{p}_{w}$ are understood to be functions of $\tilde{\Psi}$ and can be expressed instead in terms of a coordinate $x_{w}$ that identifies the location at which a streamline leaves the surface. Thus,

$$
\tilde{\rho}_{w}=\tilde{\rho}\left(x_{w}, 0\right), \quad \tilde{v}_{w}=\tilde{v}\left(x_{w}, 0\right), \quad \tilde{p}_{w}=\tilde{p}\left(x_{w}\right)
$$

where $x_{w}(\tilde{\Psi})$ is defined by

$$
\mathrm{d} \tilde{\Psi}=-\tilde{m} \mathrm{~d} x_{w}, \quad \tilde{m}=\tilde{\rho}_{w} \bar{v}_{w}
$$

The Mach number of the injected gas is also expressed as a function of $x_{w}$ :

$$
M_{w}=\alpha \bar{M}_{w}\left(x_{w}\right)
$$

From Eqs. (18) and (20), the streamline equation can be written as

$$
\begin{aligned}
\mathrm{d} y & =-\left(\frac{\gamma_{b}-1}{2}\right)^{1 / 2} \tilde{M}_{w}\left(\frac{\bar{p}}{\tilde{p}_{w}}\right)^{-1 / \gamma_{b}} \\
& \times\left\{1-\left(\frac{\tilde{p}}{\tilde{p}_{w}}\right)^{\left(\gamma_{b}-1\right) / \gamma_{\mathrm{b}}}\right\}^{-1 / 2} \mathrm{~d} x_{w}
\end{aligned}
$$

The contribution $\bar{\Delta}(x)$ to the shape of the equivalent body is found by integration over $x_{w}$, at a given $x$, from a point on 
the surface up to the zero streamline:

$$
\tilde{\Delta}(x)=\int_{\tilde{\Psi}_{w}}^{0} \frac{\mathrm{d} \tilde{\Psi}}{\tilde{\rho} \tilde{u}}=-\int_{0}^{x} \frac{\partial y}{\partial x_{w}} \mathrm{~d} x_{w}
$$

where $\tilde{\Psi}=\tilde{\Psi}_{w}(x)$, the stream function at the wall, is the inverse of $x=x_{w}(\Psi)$. The choice $M_{w}=\mathcal{O}(\alpha)$ gives $\bar{\Delta}=\mathscr{O}(1)$; i.e., for this choice the thickness of the blown-gas layer is of the same order of magnitude as the wedge thickness when $x$ $=\hat{O}(1)$.

The average mass flow per unit area equals the integral of $\rho_{w} v_{w}$ divided by the length $L$. If $\rho_{w} v_{w}$ is proportional to a power of $x$, the scaled mass flow $\tilde{m}$ can then be written in terms of a scaled average $\tilde{m}_{\text {avg }}$, which is proportional to the integrated dimensional mass flow $\dot{m}$ per unit span:

$$
\begin{gathered}
\tilde{m}=\tilde{m}_{\mathrm{avg}}\left(\frac{X_{r}}{L}\right)^{-j}(1-j) x^{-j}, \quad \tilde{m}_{\mathrm{avg}}=\frac{X_{r}}{L} \int_{0}^{L / X_{r}} \tilde{m}(x) \mathrm{d} x \\
\dot{m}=\rho_{\infty} u_{\infty} L M_{\infty} \alpha^{3}\left(\frac{\sigma_{b} \gamma_{b}}{\gamma_{\infty}}\right)^{1 / 2} \tilde{m}_{\mathrm{avg}}
\end{gathered}
$$

The mass flow $\tilde{m}$ is taken to have the same form for all $x$. In the strong-interaction region, for $x<1$, self-similarity requires that the pressure and the blowing Mach number have the form

$$
\tilde{p}=p_{1}^{(s)} x^{-1 / 2}, \quad \tilde{M}_{w}=\tilde{M}_{w 1}^{(s)} x^{-1 / 4}
$$

These conditions can be achieved, for example, if either the wall density $\tilde{\rho}_{w}$ or the wall temperature $\tilde{T}_{w}$ is constant, with the other of these quantities following a power-law variation found from the equation of state. In the weak-interaction region, the scaled pressure and the blowing Mach number are

$$
\tilde{p}=p_{0}+p_{1}^{(w)} x^{n-1}+\cdots, \quad \tilde{M}_{w}=\tilde{M}_{w 1}^{(w)} x^{-j}+\cdots
$$

It is then found from Eq. (22) that the contributions of the blown-gas layer to equivalent body shapes for the strong- and weak-interaction regions are, respectively,

$$
\begin{gathered}
\tilde{\Delta}^{(s)}=\tilde{\Delta}_{1}^{(s)} x^{3 / 4}+\cdots, \quad \tilde{\Delta}_{1}^{(s)}=\tilde{D}^{(s)} \tilde{M}_{w 1}^{(s)} \\
\tilde{\Delta}^{(w)}=\tilde{\Delta}_{1}^{(w)} x^{(3-2) / 3}+\cdots \\
\tilde{\Delta}_{1}^{(w)}=\tilde{D}^{(w)}\left(p_{0} / p_{1}^{(w)}\right)^{1 / 2} \tilde{M}_{w 1}^{(w)}
\end{gathered}
$$

and the exponents $j$ and $n$ are related by $n=(3-2 j) / 3$.

If the wall temperature $\tilde{T}_{w}$ is specified to be constant, the density $\tilde{\rho}_{w}$ and the velocity $\bar{v}_{w}$ at the surface are $\mathcal{O}\left(x^{-1 / 2}\right)$ and $\hat{O}\left(x^{-1 / 4}\right)$, respectively, for $x<<1$. The exponent $j$ appearing in $\bar{m}=\bar{v}_{w} \tilde{p} / \tilde{T}_{w}$ is $3 / 4$, and the injected mass flux is proportional to $x^{-3 / 4}$. The coefficient for the blowing Mach number in Eq. (25) is

$$
\tilde{M}_{w 1}^{(s)}=\frac{\tilde{T}_{w}^{1 / 2}}{4 p_{1}^{(s)}} \tilde{m}_{\mathrm{avg}}\left(\frac{L}{X_{r}}\right)^{3 / 4}
$$

Substitution in Eq. (27) then provides a relation between $p_{1}^{(s)}$ and $\bar{\Delta}_{1}^{(s)}$. Integration of Eq. (22) gives the constant $\bar{D}^{(s)}$ for the streamline shape in the strong-interaction region as

$$
\tilde{D}^{(s)}=\left\{2 \pi\left(\gamma_{b}-1\right)\right\}^{1 / 2} \frac{2 \gamma_{b}^{2} \Gamma\left(\frac{1}{2} \gamma_{b} /\left(\gamma_{b}-1\right)\right)}{\left(2 \gamma_{b}-1\right) \Gamma\left(\frac{1}{2} \gamma_{b} /\left(\gamma_{b}-1\right)\right)}
$$

In the weak-interaction region, again for constant wall temperature, the density and the coefficient in the blowing Mach number are

$$
\tilde{\rho}_{w}=\frac{p_{0}}{\tilde{T}_{w}}+\cdots, \quad \tilde{M}_{w 1}^{(w)}=\frac{\tilde{T}_{w}^{1 / 2}}{4 p_{0}} \tilde{m}_{\mathrm{avg}}\left(\frac{L}{X_{r}}\right)^{3 / 4}
$$

Since pressure changes are small for $x>1$, the Mach number remains small throughout the blown-gas layer, and the streamline shape is found from the incompressible form of $\mathrm{Eq}$. (22). Integration then gives the constant $\tilde{D}^{\left(w^{\prime}\right)}$ in Eq. (28) for the thickness of this layer:

$$
\mathrm{d} y=-\left(\frac{\gamma_{b} p_{0}}{2\left(\tilde{p}_{w}-\bar{p}\right)}\right)^{1 / 2} \bar{M}_{w} \mathrm{~d} x_{w} \quad \bar{D}^{(w)}=2\left(2 \gamma_{b}\right)^{1 / 2}
$$

If instead the surface density $\tilde{\rho}_{w}$ is taken to be constant, ${ }^{10}$ the exponent in $\tilde{m}=\tilde{p}_{w} \tilde{v}_{w}$ is $j=1 / 2$ and the mass flux is proportional to $x^{-1 / 2}$. It is shown in Ref. 10 that the expressions obtained for $\tilde{D}^{(s)}$ and $\tilde{D}^{(w)}$ are the same as those given by Eqs. (30) and (32).

\section{Viscous Shear Layer}

In the high-temperature separated free-shear layer, the velocity and the thermodynamic variables have the form

$$
\begin{gathered}
u / u_{\infty}=\bar{u}(x, \bar{\Psi})+\cdots, \quad p / p_{\infty}=M_{\infty}^{2} \alpha^{2} \bar{p}(x)+\cdots \\
\rho / \rho_{\infty}=\alpha^{2} \bar{\rho}(x, \bar{\Psi})+\cdots, \quad T / T_{\infty}=M_{\infty}^{2} \bar{T}(x, \bar{\Psi})+\cdots \\
h / h_{\infty}=M_{\infty}^{2} \bar{h}(x, \bar{\Psi})+\cdots
\end{gathered}
$$

where the stream function and the streamline equation are

$$
\Psi=\alpha^{2} \bar{\Psi}, \quad y_{\bar{\Psi}}=1 /(\bar{\rho} \bar{u})+\cdots
$$

If the shear layer is treated as a binary mixture of ideal gases, the equation of state is

$$
\bar{p}=\bar{\rho} R \bar{T}, \quad R=1-Y_{b}+\sigma_{b}^{-1} Y_{b}
$$

where $Y_{b}$ is the mass fraction of blown gas and $\left(1-Y_{b}\right)$ is the mass fraction of air; real-gas effects will be considered later. With each constituent modeled by the same "pointcenter-of-repulsion" potential-energy function, the transport coefficients for mass, momentum, and heat are all proportional to $\bar{T}^{\omega}{ }^{12}$ For a chemically insert mixture with negligible thermal diffusion, the concentration, momentum, and energy equations are then (e.g., Ref. 13)

$$
\begin{gathered}
Y_{b_{x}}=\frac{1}{S c}\left(K_{D} \bar{T}^{\omega} \bar{\rho} \bar{u} Y_{b \bar{\Psi}}\right)_{\bar{\Psi}} \\
\bar{u}_{x}+\frac{1}{\gamma_{\infty} \bar{\rho} \bar{u}} \frac{\mathrm{d} \bar{p}}{\mathrm{~d} x}=\left(K_{\mu} \bar{T}^{\omega} \bar{\rho} \bar{u} \bar{u}_{\bar{\Psi}}\right)_{\bar{\Psi}} \\
\bar{c}_{p} \bar{T}_{x}-\frac{1}{\bar{\rho}} \frac{\mathrm{d} \bar{p}}{\mathrm{~d} x}=\gamma_{\infty} K_{\mu} \bar{T}^{\omega} \bar{\rho} \bar{u} \bar{u}_{\Psi}^{2} \\
+\frac{\gamma_{\infty}}{\left(\gamma_{\infty}-1\right) P_{r}}\left(K_{\Lambda} \bar{T}^{\omega} \bar{\rho} \bar{u} \bar{T}_{\bar{\Psi}}\right)_{\bar{\Psi}} \\
+\frac{1}{S_{c}} K_{D} \bar{T}^{\omega}\left(\bar{c}_{P_{b}}-\bar{c}_{p_{x}}\right) \bar{\rho} \bar{u} Y_{b \bar{\Psi}} \bar{T}_{\bar{\Psi}}
\end{gathered}
$$

The specific heats appearing in Eq. (38) are

$$
\begin{gathered}
\bar{c}_{p}=\bar{c}_{p_{b}} Y_{b}+\bar{c}_{p_{x}}\left(1-Y_{b}\right) \\
\bar{c}_{p_{b}}=\frac{\sigma_{b}^{-1} \gamma_{b}}{\gamma_{b}-1}, \quad \bar{c}_{p_{x}}=\frac{\gamma_{x}}{\gamma_{\infty}-1}
\end{gathered}
$$


where $\bar{c}_{p}$ has been made nondimensional with the gas constant for undisturbed air, and the (dimensional) species specific heats $c_{p b}$ and $c_{p x}$ have been assumed independent of temperature. The Prandtl number $\mathrm{Pr}$ and Schmidt number $S c$ are defined by $\operatorname{Pr}=c_{p x} \mu_{x} / \lambda_{x}$ and $S c=\mu_{x} /\left(\rho_{x} D_{x}\right)$, where $\lambda_{x}$ is the thermal conductivity and $D_{\infty}$ is the self-diffusion coefficient, for undisturbed air. The proportionality factors in the diffusion coefficients are given by ${ }^{14}$

$$
\begin{gathered}
K_{D}=\frac{G^{-1}}{\sigma_{b} R}, \quad G=\left(\frac{2}{\sigma_{b}\left(1+\sigma_{b}\right)}\right)^{1 / 2} \\
K_{\mu}=\frac{1-Y_{b}}{1-Y_{b}+G Y_{b}}+\frac{\sigma_{b}^{1 / 2} Y_{b}}{Y_{b}+G \sigma_{b}^{3 / 2}\left(1-Y_{b}\right)} \\
K_{\lambda}=\frac{1-Y_{b}}{1-Y_{b}+G Y_{b}} \frac{E u_{a}}{E u_{\infty}}+\frac{\sigma_{b}^{-1 / 2} J Y_{b}}{Y_{b}+G \sigma_{b}^{3 / 2}\left(1-Y_{b}\right)}
\end{gathered}
$$

where $J=1$ or $J=\left(E u_{x}\right)^{-1}$ for an injected diatomic or monatomic gas, respectively. The Eucken correction $E u$ is approximated $^{15}$ by $E u_{a, x}=0.115+0.354 c_{p_{a, x}}$. With the specific heat of air assumed constant, $E u_{a}=E u_{\infty}$; otherwise $c_{p a}$ is the specific heat of air evaluated at the local temperature.

If the pressure is proportional to a power of $x$, the equations have self-similar solutions and can be reduced to ordinary differential equations. The pressure $\bar{p}$ and the similarity variable $\zeta$ are

$$
\bar{p}=p_{1} x^{q-1}+\cdots, \quad \zeta=\frac{\bar{\Psi}}{p_{1}^{1 / 2} x^{q / 2}}
$$

For strong interaction $q=1 / 2$, in agreement with Eq. (4) for the shock layer and Eq. (25) for the blown layer, and $p_{1}=$ $p_{1}^{(s)}$; for weak interaction the pressure is nearly constant, and so $q=1$ and $p_{1}=p_{0}$. Now $\bar{u}, \bar{T}$, and $Y_{b}$ are functions only of $\zeta$. The differential equations, Eqs. (36-38), become

$$
\begin{gathered}
\frac{1}{2} q \zeta Y_{b}^{\prime}=-\frac{1}{S c}\left(K_{D} R^{-1} \bar{T}^{\omega-1} \bar{u} Y_{b}^{\prime}\right)^{\prime} \\
\frac{1}{2} q \zeta \bar{u}^{\prime}-\frac{q-1}{\gamma_{\infty}} \frac{R \bar{T}}{\bar{u}}=-\left(K_{\mu} R^{-1} \bar{T}^{\omega-1} \bar{u} \bar{u}^{\prime}\right)^{\prime} \\
\frac{\bar{c}_{p}}{2 \gamma_{\infty}} q \zeta \bar{T}^{\prime}+\frac{q-1}{\gamma_{\infty}} R \bar{T}=-K_{\mu} R^{-1} \bar{T}^{\omega-1} \bar{u} \bar{u}^{\prime 2} \\
-\frac{1}{\left(\gamma_{\infty}-1\right) P r}\left(K_{\lambda} R^{-1} \bar{T}^{\omega-1} \bar{u} \bar{T}^{\prime}\right)^{\prime} \\
-\frac{1}{\gamma_{\infty} S c} K_{D} R^{-1} \bar{T}^{\omega-1} \bar{u}\left(\bar{c}_{p_{b}}-\bar{c}_{p_{x}}\right) Y_{b}^{\prime} \bar{T}^{\prime}
\end{gathered}
$$

Numerical integration of these equations is carried out for $\zeta_{l}$ $<\zeta<\infty$, where $\zeta_{l}<0$ and $\left|\zeta_{l}\right| p_{1}^{1 / 2} x^{q / 2}$ is the (scaled) amount of mass entrained in the part of the shear layer below the zero streamline. The boundary conditions as $\zeta \rightarrow \infty$ require that $\bar{u}$ $\rightarrow 1, \bar{T} \rightarrow 0$, and $Y_{b} \rightarrow 0$, and as $\zeta \rightarrow \zeta_{l}$ it is required that $\bar{u}$ $\rightarrow 0, \bar{T} \rightarrow 0$, and $Y_{b} \rightarrow 1$; moreover, the asymptotic form as $\zeta \rightarrow \zeta_{l}$ is known, and the solutions for $\bar{u}$ and $\bar{T}$. must approach zero in the proper way. The computational scheme is a continuation method in which the value of $\zeta$ at the lower boundary is taken to be a small negative number that is gradually increased in magnitude until the correct asymptotic behavior is achieved. ${ }^{11}$ Since the numerical scheme in Ref. 11 does not appear to converge for $q=1$, the weak-interaction solutions are found by linear extrapolation from solutions computed for $q$ close to 1 (e.g., $q=0.98$ and 0.99 ).

The streamline equation is found from Eq. (34) as

$$
y_{\zeta}=p_{1}^{-1 / 2} x^{1-q / 2} R \bar{T} / \bar{u}
$$

For strong and weak interaction, respectively, the shear-layer thicknesses are

$$
\begin{array}{ll}
\bar{\Delta}^{(s)}(x)=\bar{\Delta}_{1}^{(s)} x^{3 / 4}+\cdots, & \bar{\Delta}_{1}^{(s)}=\left(p_{1}^{(s)}\right)^{-1 / 2} \bar{D}^{(s)} \\
\bar{\Delta}^{(w)}(x)=\bar{\Delta}_{1}^{(w)} x^{1 / 2}+\cdots, & \bar{\Delta}_{1}^{(w)}=p_{0}^{-1 / 2} \bar{D}^{(w)}
\end{array}
$$

where $\bar{D}^{(s)}$ and $\bar{D}^{(w)}$ are numbers found by integrating the appropriate solutions for $R \bar{T} / \bar{u}$ from $\zeta_{l}$ to $\infty$. These integrals exist since $\bar{T} \rightarrow 0$ sufficiently rapidly as $\zeta \rightarrow \infty$, as can be seen by deriving the asymptotic form for $\bar{T}$. For strong interaction $\alpha \bar{\Delta}^{(s)}(x)=\mathcal{O}\left(\alpha x^{3 / 4}\right)$, and the shear-layer thickness remains small in comparison with $x$ when $x>\alpha^{4}$; i.e., as anticipated, the boundary-layer approximation fails in the merged-layer regime $x=\mathscr{O}\left(\alpha^{4}\right)$.

The analysis may be extended to include real-gas effects that occur at high temperatures. For air, the specific heats begin to vary when $T \geqslant 600 \mathrm{~K}$, and $\mathrm{O}_{2}$ begins to dissociate when $T \geq 2000-2500 \mathrm{~K}$, for $p \approx 0.01-1$ atm. Self-similar solutions may still be computed when the gas is chemically reactive provided the pressure is nearly constant, as in the weak-interaction region, and chemical equilibrium will be closely approached sufficiently far downstream.

To provide a measure of the extent by which the ideal-gas results will change, the analysis of the weak-interaction region is repeated for the case of air injection, with air treated as a real gas in chemical equilibrium. Following Hansen's analysis, ${ }^{16}$ it is assumed that, for a given pressure, the peak temperature remains low enough that, to a good approximation, only the dissociation of oxygen occurs. (For pressures of the order of $0.1 \mathrm{~atm}$, this assumption limits the temperature to values below approximately $4000 \mathrm{~K}$; the limit increases with increasing pressure.) Air is then composed of $\mathrm{N}_{2}, \mathrm{O}_{2}$, and $\mathrm{O}$, with the mass fraction of $O$ given by $Y_{\mathrm{O}}=2(16 / 29) \varepsilon$, where $16 / 29$ is the approximate ratio of the molecular mass of $O$ to that of undissociated air. The fraction $\varepsilon$ of molecules that dissociate into oxygen atoms may be written ${ }^{16}$

$$
\varepsilon=\left\{-0.8+\left[0.64+0.8\left(1+4 p / K_{p}^{2}\right)\right]^{1 / 2}\right\} /\left(1+4 p / K_{p}^{2}\right)
$$

The equilibrium constant $K_{p}$ for $\frac{1}{2} \mathrm{O}_{2} \rightleftarrows \mathrm{O}$ is given to within $4 \%$ of the values listed in Ref. 17 over the temperature range $1500-4000 \mathrm{~K}$ (with $K_{p}^{2}$ and $p$ expressed in atmospheres) by

$$
K_{p}=40.68 T^{1 / 2} \exp \{-29,685 / T\}
$$

where $T$ is expressed in degrees Kelvin; the quantity 29,685 is, in degrees Kelvin, the formation enthalpy of $\mathrm{O}$ at absolute zero divided by the universal gas constant. With $R=1+\varepsilon$, the equation of state remains as written in Eq. (35), ${ }^{16}$ and the scaled enthalpy $\bar{h}$ is approximately related to the temperature by

$$
\bar{h}=\bar{T}\left(1+\frac{2}{7} \frac{3390 / T}{\exp (3390 / T)-1}+\frac{4}{7} \varepsilon \frac{29,685}{T}\right)
$$

where the sensible enthalpy of $\mathrm{O}_{2}$ has been equated with that of $\mathrm{N}_{2}$, and sensible enthalpies in the term proportional to $\varepsilon$ have been neglected in comparison with the formation enthalpy of $O$. Since only air injection is considered, the species conservation Eq. (44) may be dropped. If dissociated air is approximated as a binary mixture of "air molecules" and $\mathrm{O}$ atoms in evaluating the viscous fluxes, then treatment of the transport properties as before leaves the momentum equation (with $q=1$ ) unchanged, and the energy equation may be written more conveniently in the form

$$
\begin{aligned}
& -\frac{1}{2} \zeta \bar{h}^{\prime}=\left(\gamma_{\infty}-1\right) K_{\mu} \bar{T}^{\omega-1} R^{-1} \bar{u} \bar{u}^{\prime 2} \\
& +1 / \operatorname{Pr}\left\{\left(K_{\lambda}+K_{\lambda D}\right) \bar{T}^{\omega-1} R^{-1} \bar{u} \bar{T}^{\prime}\right\}^{\prime}
\end{aligned}
$$


The proportionality factor $K_{\lambda D}$ for energy transfer by mass diffusion is given by

$$
\begin{aligned}
K_{\lambda D} & =\frac{\gamma_{\infty}-1}{\gamma_{\infty}} \frac{\operatorname{Pr}}{S c} K_{D} \frac{29,685}{T} \\
& \times\left[\frac{2(1 / 2+29,685 / T)}{1 / \varepsilon+0.5 /(0.2-\varepsilon)-0.5 /(1+\varepsilon)}\right]
\end{aligned}
$$

where sensible enthalpies have been neglected, and the term in brackets [similar to Hansen's ${ }^{16}$ Eq. (43a)] is proportional to $T \partial Y_{\mathrm{O}} / \partial T$. The quantity $K_{\mu}$ is taken to be unity (considering the current level of approximation), and $K_{\lambda}, K_{D}$ are given by Eqs. (40), (42), respectively, with $\sigma_{b}=16 / 29, Y_{b}=Y_{\mathrm{O}}$, and $J=1$; the Eucken correction $E u_{a}$ for "air molecules" is evaluated with $\bar{c}_{p_{a}}=7 / 2+\{3390 /(2 T)\} / \sinh ^{2}\{3390 /(2 T)\}$, the nondimensional specific heat of $\mathrm{N}_{2} \cdot{ }^{16}$ The boundary conditions remain the same as before, and Eqs. (45) and (53) are solved following the same numerical procedure as described earlier; the pressure-gradient term, $-\left\{\left(\gamma_{x}-1\right) / \gamma_{x}\right\}(q-1) R \bar{T}$, is added to the left-hand side of Eq. (53) for computational purposes, with $q$ approaching 1 . Unlike the ideal-gas case, the frestream conditions and wedge angle must be specified before computing a solution since real-gas behavior is explicitly dependent on dimensional temperature and pressure.

\section{Results}

The location of a streamline in the shock layer can be expressed by writing the integral of the streamline equation in three parts:

$$
y=\rho_{\infty} u_{\infty} \int_{\Psi_{w}}^{\alpha^{2} \bar{\Psi}_{l} l} \frac{\mathrm{d} \Psi}{\rho u}+\rho_{\infty} u_{\infty} \int_{\alpha^{2} \bar{\Psi}_{l}}^{\alpha^{2} \bar{\Psi}_{u}} \frac{\mathrm{d} \Psi}{\rho u}+\rho_{\infty} u_{\infty} \int_{\alpha^{2} \bar{\Psi}_{u}}^{\Psi} \frac{\mathrm{d} \Psi}{\rho u}
$$

where $u$ becomes small as $\bar{\Psi}$ decreases toward $\bar{\Psi}_{l} ; \Psi_{w}=$ $\sigma\left(M_{\infty} \alpha^{2}\right)$ is the value of $\Psi$ at the wall; and $\bar{\Psi}_{\omega}$ is still arbitrary. Then

$$
y=\int_{\tilde{\Psi}_{w}}^{b \bar{\Psi} / / M_{\star}} \frac{\mathrm{d} \tilde{\Psi}}{\tilde{\rho} \tilde{u}}+\int_{\bar{\Psi}_{l}}^{\bar{\Psi}_{u}} \frac{\mathrm{d} \bar{\Psi}}{\bar{\rho} \bar{u}}+\int_{\alpha^{2} \bar{\Psi}_{u}}^{\hat{\Psi}} \frac{\mathrm{d} \hat{\Psi}}{\hat{\rho} \hat{u}}
$$

where $b=\left(\sigma_{b} \gamma_{b} / \gamma_{\infty}\right)^{-1 / 2}$. For $\alpha<<1$ and $\bar{\Psi}_{u}>>1$ such that $\alpha^{2} \bar{\Psi}_{u}<<1$, the first integral approaches $\tilde{\Delta}(x)$ as defined by Eq. (23); the second integral approaches a finite value $\bar{\Delta}(x)$ since $\bar{\rho} \rightarrow \infty$ rapidly enough as $\bar{\Psi} \rightarrow \infty$; and the third integral approaches zero if also $\Psi \rightarrow 0$. Then the left-hand side approaches the equivalent body shape $y=\Delta(x)$, and so Eq. (56) approaches the first of Eqs. (3). For strong and weak interactions, respectively, the coefficients in the equivalent body shapes defined by Eqs. (4) and (9) include contributions from both the thin layer of blown gas and the viscous free shear layer:

$$
\Delta_{1}^{(s)}=\tilde{\Delta}_{1}^{(s)}+\bar{\Delta}_{1}^{(s)}, \quad \Delta_{1}^{(w)}=\tilde{\Delta}_{1}^{(w)}+\bar{\Delta}_{1}^{(w)}
$$

The pressures are found in terms of these quantities from Eqs. (5), (27), and (48) for strong interaction, and from Eqs. (14), (28), and (49) for weak interaction. As indicated by the notation, the pressures in the blown-gas layer and the viscous layer are equal to the pressure in the outer inviscid flow evaluated at $y=\Delta(x)$ if $x<<1$ and at $y=0$ if $x>>1$.

The constants $\hat{D}^{(s)}$ and $\hat{D}^{(w)}$ introduced in Eqs. (5) and (14) are given in Table 1 for $\gamma_{\infty}=1.4$ and, in the case of $D^{(w)}$, for $n=1 / 2\left(\tilde{T}_{w}=\right.$ const $)$ or $n=2 / 3\left(\tilde{\rho}_{w}=\right.$ const $)$ and a representative value $M_{\nsim} \alpha=1$. The constants $\tilde{D}^{(s)}$ and $\tilde{D}^{(w)}$ defined for the blown-gas layer in Eqs. (27) and (28) are functions only of $\gamma_{b}$; the values for $\gamma_{b}=7 / 5$ and $\gamma_{b}=5 / 3$ found from the solutions in Eqs. (30) and (32) are shown in Table 2 . Some ideal-gas results for the viscous shear layer are listed in Table 3 for injected gases having a wide range of molecular weights. The constants $\bar{D}^{(s)}$ and $\bar{D}^{(w)}$ defined in Eqs. (48) and (49) are shown, along with the lower limit $\zeta_{l}$ for the similarity variable in both the strong- and weak-interaction solutions. The scaled maximum temperature $\bar{T}_{\max }$ is seen to be a small number in each case; to find the actual maximum temperature in the shear layer, the value of $\bar{T}_{\max }$ is to be multiplied by $M_{\infty}^{2} T_{\infty}$. For example, with air injection the maximum in the strong-interaction region is about $0.03 M_{\infty}^{2} T_{\infty}$, implying that significant levels of $\mathrm{O}_{2}$ dissociation (which, as noted earlier, begin when the local temperature is about $2000-2500 \mathrm{~K}$ for $p \geqslant 0.01-1 \mathrm{~atm}$ ) will not occur until $M_{\infty} \geqslant 15-17$ if $T_{\infty} \approx 300$ $\mathrm{K}$; for helium injection, cooling of the viscous layer increases this Mach number to $M_{\infty} \geq 26-29$. The ideal-gas results are understood to be applicable when the Mach number is low enough (or the shear layer thin enough) that dissociation effects are not appreciable. A comparison between the idealand real-gas results for the shear layer is presented in Table 4 for the case of air injection on a 6-deg wedge at an altitude of $30 \mathrm{~km}$ with $M_{\infty}=15$ or 20 . It may be seen that the realgas analysis yields values for the maximum temperature $T_{\max }$ that are lower than the ideal-gas values, as would be expected; the values of $\bar{D}^{(w)}$, which are proportional to the layer thickness, are likewise reduced. For the conditions considered, however, real-gas effects do not greatly alter the values of $\bar{D}^{(w)}$ and $\zeta_{l} ;$ for example, there is only a $5 \%$ decrease in $\bar{D}^{(w)}$ when $M_{\infty}=20$. The differences would of course be greater if higher Mach numbers and temperatures had been considered.

If the density of the gas at the wall is constant, the pressures are found, using the definitions of $M_{w}$ and $\tilde{m}_{\text {avg }}$, in an explicit
Table 1 Shock-layer data

\begin{tabular}{|c|c|c|c|c|c|c|c|c|}
\hline \multirow[b]{2}{*}{ Injectant } & \multirow[b]{2}{*}{$\gamma_{b}$} & \multirow[b]{2}{*}{$\sigma_{b}$} & \multicolumn{3}{|c|}{ Strong interaction, $q=1 / 2$} & \multicolumn{3}{|c|}{ Weak interaction, $q=1$} \\
\hline & & & $\overline{D^{(s)}}$ & $-\xi_{1}$ & $\bar{T}_{\max }$ & $\bar{D}^{(w)}$ & $-\zeta_{1}$ & $\bar{T}_{\max }$ \\
\hline$\overline{\mathrm{H}_{2}}$ & $7 / 5$ & 0.0690 & 0.1103 & 0.9623 & 0.0047 & 0.1259 & 0.6107 & $\overline{0.0079}$ \\
\hline $\mathrm{He}$ & $5 / 3$ & 0.1379 & 0.1796 & 1.4262 & 0.0098 & 0.2157 & 0.8101 & 0.0168 \\
\hline $\mathrm{Ne}$ & $5 / 3$ & 0.6897 & 0.3515 & 3.2133 & 0.0258 & 0.4384 & 1.6391 & 0.0437 \\
\hline Air & $7 / 5$ & 1.0000 & 0.3669 & 3.8154 & 0.0280 & 0.4331 & 1.9665 & 0.0447 \\
\hline $\mathrm{Ar}$ & $5 / 3$ & 1.3793 & 0.4604 & 4.5670 & 0.0356 & 0.5896 & 2.1818 & 0.0594 \\
\hline
\end{tabular}

\begin{tabular}{lcc}
\hline \hline Case & $\hat{D}^{(s)}$ & $\hat{D}^{(w) \mathrm{a}}$ \\
\hline$\tilde{T}_{w}=$ const $^{\mathrm{b}}$ & 0.945 & 0.5675 \\
$\tilde{\rho}_{w}=$ const $^{\mathrm{c}}$ & 0.945 & 0.4150 \\
\hline \hline aEquation (14) with $M_{\mathrm{x}} \alpha=1$. & \\
$\mathrm{b}_{n}=1 / 2$. & \\
$c_{n}=2 / 3$. &
\end{tabular}

Table 2 Data for blown-gas layer

\begin{tabular}{lcc}
\hline \hline$\gamma_{b}$ & $\tilde{D}^{(s) \mathrm{b}}$ & $\tilde{D}^{(w) \mathrm{c}}$ \\
\hline $7 / 5$ & 3.501 & 3.347 \\
$5 / 3$ & 3.604 & 3.651 \\
\hline \hline "Either $T_{w}$ or $\rho_{w}$ is a constant. & \\
"Equation (30). & \\
'Equation (32). &
\end{tabular}

Table 3 Shear-layer data 
Table 4 Comparison between ideal- and real-gas results for shear layer ${ }^{\mathrm{a}}$

\begin{tabular}{|c|c|c|c|c|c|c|}
\hline \multirow[b]{2}{*}{$M_{\text {× }}$} & \multicolumn{3}{|c|}{ Ideal } & \multicolumn{3}{|c|}{ Real } \\
\hline & $\bar{D}^{(n)}$ & $-\zeta_{i}$ & $T_{\max }, \mathrm{K}$ & $\bar{D}^{(w)}$ & $-\zeta_{i}$ & $T_{\max }, \mathrm{K}$ \\
\hline $15^{b}$ & 0.4331 & 1.9665 & 2326 & 0.4099 & 1.9825 & 2082 \\
\hline $20^{\mathrm{c}}$ & 0.4331 & 1.9665 & 4135 & 0.4131 & 1.9949 & 3662 \\
\hline
\end{tabular}

${ }^{\mathrm{a}} \gamma_{\mathrm{x}}=1.4, \omega=0.75, \operatorname{Pr}=0.72, S c=0.74, \alpha=6 \mathrm{deg}$, and $z=30 \mathrm{~km}\left(T_{x}=231.2 \mathrm{~K}, p_{x}=0.1174\right.$

atm).

${ }^{\mathrm{b}} M_{\times}^{2} T_{x}=52,020 \mathrm{~K}$ and $p=0.07131 \mathrm{~atm}$

${ }^{c} M_{x}^{2} T_{x}=92,480 \mathrm{~K}$ and $p=0.10991 \mathrm{~atm}$

form equivalent to the results of Ref. 10:

$$
\begin{aligned}
& p_{1}^{(s)}=\frac{\bar{D}^{(s)}}{\hat{D}^{(s)}}+\frac{\tilde{D}^{(s)}}{\hat{D}^{(s)}} \frac{1}{2 \tilde{\rho}_{w}^{1 / 2}} \tilde{m}_{\mathrm{avg}}\left(\frac{L}{X_{r}}\right)^{1 / 2} \\
& p_{1}^{(w)}=\left(\frac{\tilde{D}^{(w)}}{2 \hat{D}^{(w)}} \bar{m}_{\mathrm{avg}}\right)^{2 / 3} \frac{1}{\tilde{\rho}_{w}^{1 / 3}}\left(\frac{L}{X_{r}}\right)^{1 / 3}
\end{aligned}
$$

In this case, the viscous shear layer for $x \gg 1$ has thickness $\mathcal{O}\left(x^{1 / 2}\right)$ that is of higher order than the blown-layer thickness, which is $O\left(x^{2 / 3}\right)$, and so the shear layer does not affect the first approximation for the pressure. If, instead, the temperature of the gas at the wall is constant, the thickness of the viscous and blown layers are of the same order of magnitude for weak interaction as well as for strong interaction. Now the pressures are obtained from solution of the cubic equation

$$
\left(P^{1 / 2}\right)^{3}-3\left(P^{1 / 2}\right)-2 Q=0
$$

where $P$ and $Q$ are defined for strong interaction by

$$
\begin{gathered}
p_{1}^{(s)}=\frac{1}{3} \frac{\bar{D}^{(s)}}{\hat{D}^{(s)}} P \\
\frac{\tilde{D}^{(s)}}{\hat{D}^{(s)}} \frac{\tilde{T}_{w}^{1 / 2}}{4}\left(\frac{L}{X_{r}}\right)^{3 / 4} \tilde{m}_{\text {avg }}=2\left(\frac{\bar{D}^{(s)}}{3 \hat{D}^{(s)}}\right)^{3 / 2} Q
\end{gathered}
$$

and for weak interaction by

$$
\begin{gathered}
p_{1}^{(w)}=\frac{1}{3 p_{0}^{1 / 2}} \frac{\bar{D}^{(w)}}{\hat{D}^{(w)}} P \\
\frac{\tilde{D}^{(w)}}{p_{0}^{1 / 2} \hat{D}^{(w)}} \frac{\bar{T}_{w}^{q / 2}}{4}\left(\frac{L}{X_{r}}\right)^{3 / 4} \tilde{m}_{\mathrm{avg}}=2\left(\frac{\bar{D}^{(w)}}{3 p_{0}^{1 / 2} \hat{D}^{(w)}}\right)^{3 / 2} Q
\end{gathered}
$$

The relevant root of the cubic equation is found from

$$
\begin{aligned}
& Q \leq 1: \\
& \quad P^{1 / 2}=2 \cos \phi, \quad \cos 3 \phi=Q, \quad 0 \leq \phi \leq \pi / 2 \\
& Q \geq 1: \\
& \quad P^{1 / 2}=2 \cosh \chi, \quad \cosh 3 \chi=Q
\end{aligned}
$$

It is seen that $P=3$ when $Q=0$ and that $P$ increases monotonically as $Q$ increases. For $Q$ sufficiently large ( $\gtrsim 16$ for less than $10 \%$ fractional error), $P \approx(2 Q)^{2 / 3}$. This may be regarded as an inviscid-flow limit, since $Q$ is proportional to $\left(L / X_{r}\right)^{3 / 4} \bar{m}_{\text {avg }}$ and $L / X_{r}$ is proportional to the Reynolds number $R e=u_{x} L / \nu_{x} ;$ thus, for fixed values of the other parameters, large $Q$ implies that $R e$ and/or the blowing rate $\tilde{m}_{\text {avg }}$ are large, and that the viscous-layer thickness may therefore be neglected.

The pressures for $x>1$ and $x<1$ have the forms given by Eqs. (25) and (26), respectively. Because of self-similarity, these require solving only ordinary differential equations, whereas a solution for $x=O(1)$ would be much more difficult to obtain and is not attempted here. Instead, since the numerical values of $p_{1}^{(s)}$ and $p_{1}^{(w)}$ are not greatly different, interpolation between the strong- and weak-interaction results would be expected to provide an approximate representation that is fairly accurate. The interpolation formula chosen is

$$
\begin{gathered}
\tilde{p}-p_{0}=r(x) p_{1}^{(s)} x^{-1 / 2}+\{1-r(x)\} p_{1}^{(w)} x^{n-1} \\
r(x)=\frac{1}{\cosh \left(A x^{1 / 2}\right)}, \quad A=\cosh ^{-12}
\end{gathered}
$$

For $x<1, r(x) \approx 1$ and Eq. (25) for strong interaction is recovered; for $x>>1, r(x) \approx 0$ and Eq. (26) for weak interaction is recovered; and $A$ is defined such that $r(x)=1 / 2$ when $x=1$. [The definition of $r(x)$ differs slightly from that in Ref. 10.] The interpolated pressure is shown by the solid curves in Fig. 3 for air injection at three different blowing rates with $\tilde{T}_{w}=$ const. The differences from the asymptotic solutions derived for $x \gg 1$ and $x<<1$ are seen to remain small, even if the asymptotic solutions are used (incorrectly) at $x=1$.

The increase $\Delta F$ in the (dimensional) normal force above the inviscid-flow value is found by integration of Eq. (65):

$$
\begin{aligned}
& \frac{\Delta F}{\left(M_{\infty} \alpha\right)^{2} p_{\infty} L}=2 \frac{X_{r}}{L}\left\{p_{1}^{(s)} I_{1 / 2}\right. \\
&\left.+p_{1}^{(w)}\left[\frac{1}{2 n}\left(\frac{L}{X_{r}}\right)^{n}-I_{n}\right]\right\}+\cdots \\
& I_{1 / 2}=\frac{1}{A} \tan ^{-1}\left\{\sinh \left(A\left(L / X_{r}\right)^{1 / 2}\right)\right\}
\end{aligned}
$$

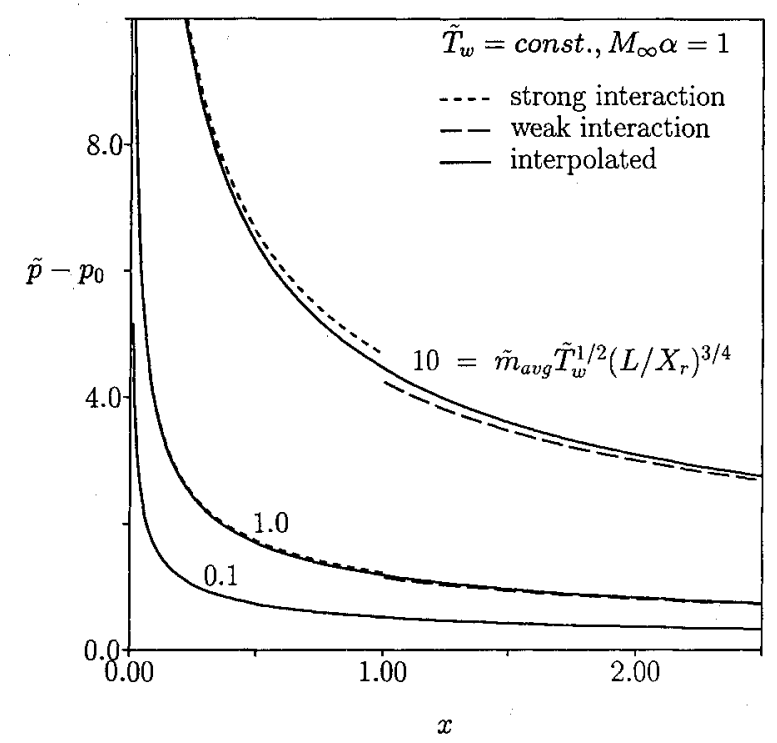

Fig. 3 Surface pressure $\tilde{p}-p_{0}$ vs $x$ for various rates of air injection. 


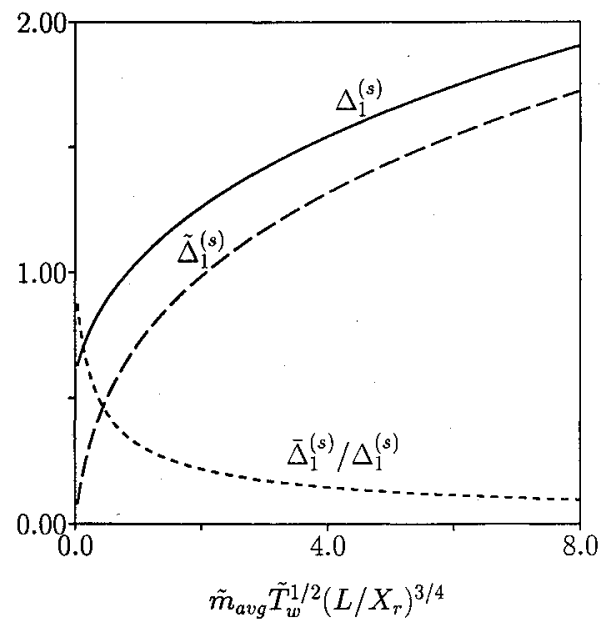

Fig. 4 Strong-interaction solutions for scaled total displacement thickness $\Delta_{1}^{(s)}$, thickness $\tilde{\Delta}_{1}^{(s)}$ of blown-gas layer, and the ratio of shearlayer to total-displacement thickness $\bar{\Delta}_{1}^{(s)} / \Delta_{1}^{(s)}$ vs blowing-rate parameter $\bar{m}_{\text {avg }} \bar{T}_{w}^{1 / 2}\left(L / X_{r}\right)^{3 / 4}$ for air injection with constant wall temperature.

$$
I_{n}=\int_{0}^{\left(L / X_{Y}\right)^{1 / 2}} s^{2 n-1} \operatorname{sech}(A s) \mathrm{d} s
$$

where $n=2 / 3$ if $\tilde{\rho}_{w}$ is constant and $n=1 / 2$ if $\tilde{T}_{w}$ is constant.

When the blowing rate is sufficiently large, for given values of the other parameters, the viscous-layer thickness is small relative to the total displacement thickness and can be neglected in a first approximation. The displacement effect is then caused entirely by the layer of blown gas, and is described by the inviscid-flow model of Cole and Aroesty. ${ }^{8}$ The approach to this limiting case is illustrated in Fig. 4, where the scaled totaldisplacement thickness $\Delta_{1}^{(s)}$, scaled blown-layer thickness $\tilde{\Delta}_{1}^{(s)}$, and the ratio of viscous-layer to total-displacement thickness $\bar{\Delta}_{1}^{(s) / \Delta_{1}^{(s)}}$ are plotted against the scaled blowing rate $\tilde{m}_{\mathrm{avg}}(L /$ $\left.X_{r}\right)^{3 / 4} \tilde{T}_{w / 2}^{1 / 2}$ for air injection at constant wall temperature. As the blowing rate increases, it is seen that $\bar{\Delta}_{1}^{(s)} / \Delta_{1}^{(s)}$ decreases, falling to less than $10 \%$ for $\tilde{m}_{\mathrm{avg}}\left(L / X_{r}\right)^{3 / 4} \tilde{T}_{w}^{1 / 2} \geq 7.2$.

It should also be noted in Fig. 4 that the solutions do not remain valid if $\tilde{m}_{\text {avg }}\left(L / X_{r}\right)^{3 / 4} \tilde{T}_{w}^{1 / 2}$ is taken to be too small. It is assumed in the present theory that the blowing rate is large enough to generate an inviscid blown layer with a thickness at least of the same order of magnitude as that of the viscous free shear layer, a condition that is not met if $\tilde{m}_{\text {avg }}(L /$ $\left.X_{r}\right)^{3 / 4} \tilde{T}_{w}^{1 / 2} \rightarrow 0$. (A somewhat weaker assumption can be shown to suffice, namely that the amount of injected mass is large in comparison with the mass in the viscous layer.) For the results presented below, the blowing rate has been chosen arbitrarily to be large enough that $\tilde{\Delta}^{(s)} \geq \bar{\Delta}^{(s)}$; for air injection this implies $\tilde{m}_{\text {avg }}\left(L / X_{r}\right)^{3 / 4} \tilde{T}_{w}^{1 / 2} \geq 0.34$.

In Fig. 5, the scaled change in force $\Delta F\left(L / X_{r}\right)^{1 / 2}$ $\left(M_{\infty}^{2} \alpha^{2} p_{\infty} L\right)$ is plotted vs the scaled total mass flow rate $\dot{m}\left(T_{w} /\right.$ $\left.T_{\infty}\right)^{1 / 2}\left(L / X_{r}\right)^{3 / 4} /\left(M_{\infty} \alpha^{3} \rho_{\infty} u_{\infty} L\right)$ for various cases, all with constant wall temperature. Two limiting cases are shown for air as the injectant. For $L / X_{r} \rightarrow 0$, the strong-interaction limit, the viscous-layer thickness is so large compared to the wedge thickness that the magnitude of $M_{x} \alpha$ is irrelevant, and the force change depends on $p_{1}^{(s)}$. For $L / X_{r} \rightarrow \infty$ and either $M_{x} \alpha$ $\rightarrow 0$ or $M_{\infty} \alpha \rightarrow \infty$, the force change depends on $p_{1}^{(w)}$; the larger force change is found for $M_{\infty} \alpha \rightarrow \infty$ and the smaller for $M_{\infty} \alpha$ $\rightarrow 0$, as might be expected since for $M_{x} \alpha \rightarrow 0$ the wedge thickness tends to zero in comparison with the total thickness of the region of disturbed flow between the wedge surface and the shock wave.

Also shown in Fig. 5 is a comparison between air and helium as injectants, for $L / X_{r}=1$ and $M_{\infty} \alpha=1$, again for $\tilde{T}_{w}=$ const. As in the inviscid-flow case considered by Cole and Aroesty, ${ }^{8}$ it is seen that strong blowing of a gas lighter than

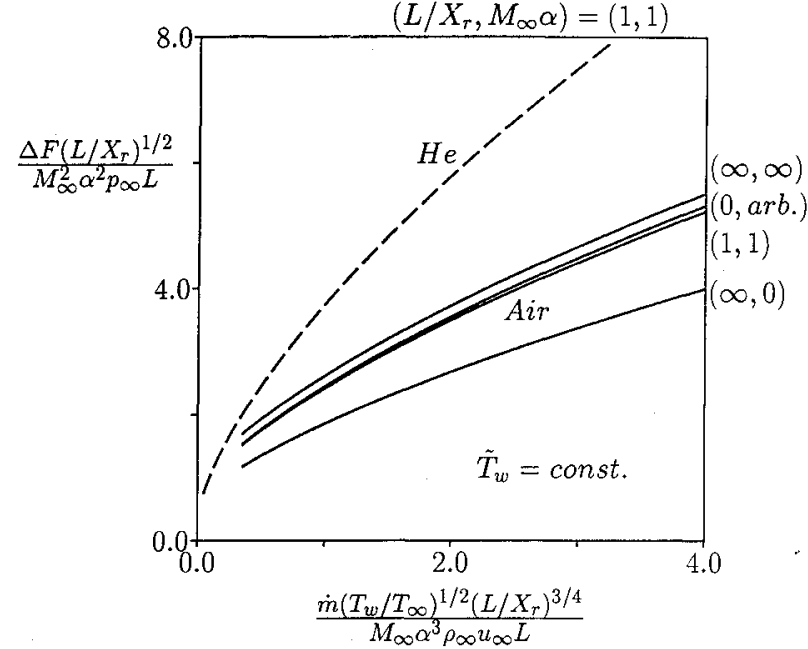

Fig. 5 Scaled dimensionless force change $\Delta F\left(L / X_{r}\right)^{1 / 2} /\left(M_{x}^{2} \alpha^{2} p_{x} L\right)$ vs scaled dimensionless mass flow rate $\dot{m}\left(T_{v} / T_{\propto}\right)^{1 / 2}\left(L / X_{r}\right)^{3 / 4} /\left(M_{\propto} \alpha^{3} \rho_{x} u_{\infty} L\right)$ for various cases.

air, in this case helium, yields higher induced surface pressures and thus larger force changes for a fixed mass flow than when air (or a gas heavier than air) is the injectant. Interestingly, this trend appears to become reversed when the strength of the blowing decreases. Introduction of a light gas increases the specific heat for the mixture and thus cools the viscous layer, as indicated by the maximum temperatures listed in Table 3. The average density is therefore increased and the transport coefficients are decreased. As a result, the thickness of the viscous layer is reduced and thus the force changes are also reduced. The curves for air and helium in Fig. 5 would therefore be expected to cross if they were extended to smaller values of mass flow.

To establish numerical orders of magnitude for the change in force and the associated mass flow rate of the injected gas, dimensional values of $\Delta F$ and $\dot{m}$ for $T_{w}=$ const have been computed for a range of parameter values and for various injectants, using the properties of the standard atmosphere. The solutions depend on several parameters that may be divided conveniently into three groups. Parameters related to flight régime are $M_{\infty}$ and altitude $z$; those characterizing wedge geometry are $\alpha$ and $L$; and those representing properties of the injected mass are $\dot{m}, T_{w}, \sigma_{b}$, and $\gamma_{b}$. (The solutions also depend on $\omega$, the exponent in the viscosity law, taken here as $3 / 4$.) Air injection on a wedge having length $L=3 \mathrm{~m}$ and half-angle $\alpha=6 \mathrm{deg}$, for $M_{\infty}=15$ and $z=30 \mathrm{~km}$, is selected as a baseline case in each part of Fig. 6, where $M_{\infty}$ and $z$ (Fig. 6a), $\alpha$ and $L$ (Fig. 6b), and injectant type (Fig. 6c) have been varied. Note that large changes in force per unit span of order $1000 \mathrm{~N} / \mathrm{m}(68.5 \mathrm{lb} / \mathrm{ft})$ are achieved with seemingly moderate injection rates per unit span of order $0.1 \mathrm{~kg} / \mathrm{m}-\mathrm{s}(0.067 \mathrm{lbm} /$ $\mathrm{ft}-\mathrm{s})$.

In Fig. 6a, it is seen that, for given injectant rates, the force changes increase with flight Mach number at a given altitude and decrease with increasing altitude at a given flight Mach number, as expected. Also presented is a comparison between the ideal-gas and real-gas results for Mach 15 and 20, where differences between the solutions are seen to be small. (Since the real-gas analysis was for weak interaction only, the realgas curves were computed using the ideal-gas results for the strong-interaction region, which extends over a negligibly small portion of the wedge for the conditions considered.) As shown in Fig. 6b, at a given flight Mach number and altitude, the force changes increase with wedge angle for a given length and increase with length for a given wedge angle. Finally, in Fig. $6 \mathrm{c}$, it is shown that the force change increases with decreasing molecular weight of the injected gas, at higher injectant mass flow rates. At lower injection rates, the opposite 
effect occurs and the crossover of the curves anticipated above is seen for argon, air, and neon. It is also observed that the crossover occurs at lower injectant rates as the molecular weight of injectant decreases. For all of the calculations in Fig. 6, the wall temperature $T_{w}$ is constant.

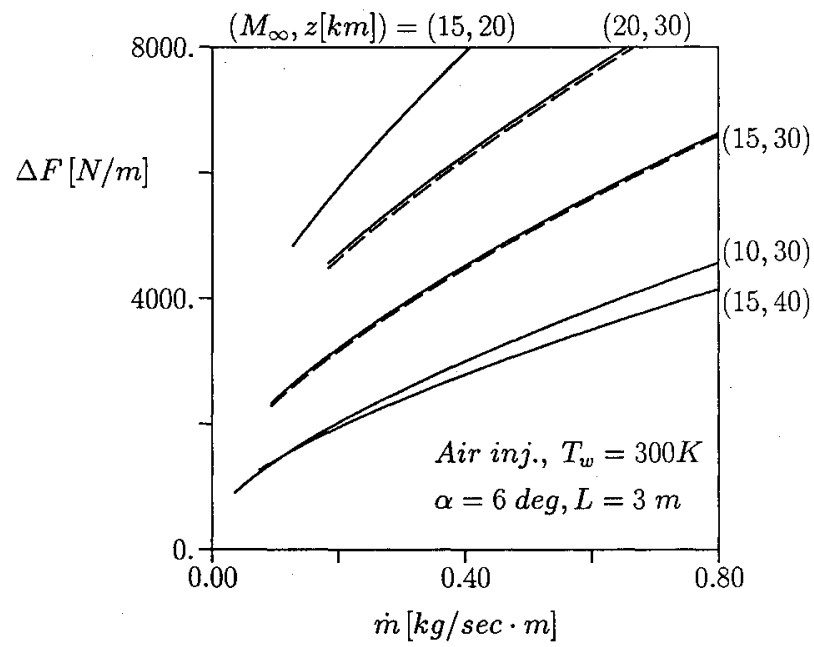

Fig. 6a Force change $\Delta F$ vs mass flow rate $\dot{m}$ for various flight regimes. (Dashed lines are real-gas results.)

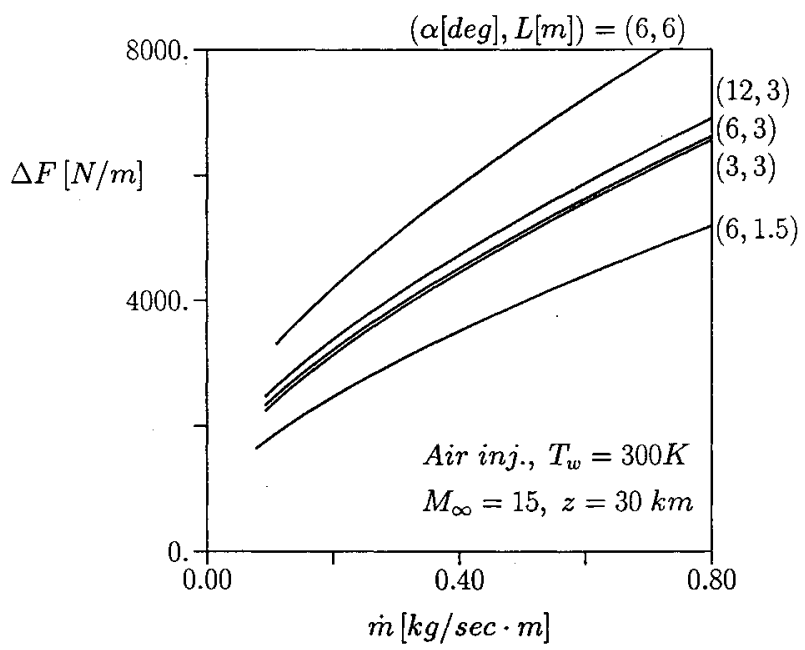

Fig. 6b Force change $\Delta F$ vs mass flow rate $\dot{m}$ for various wedge geometries.

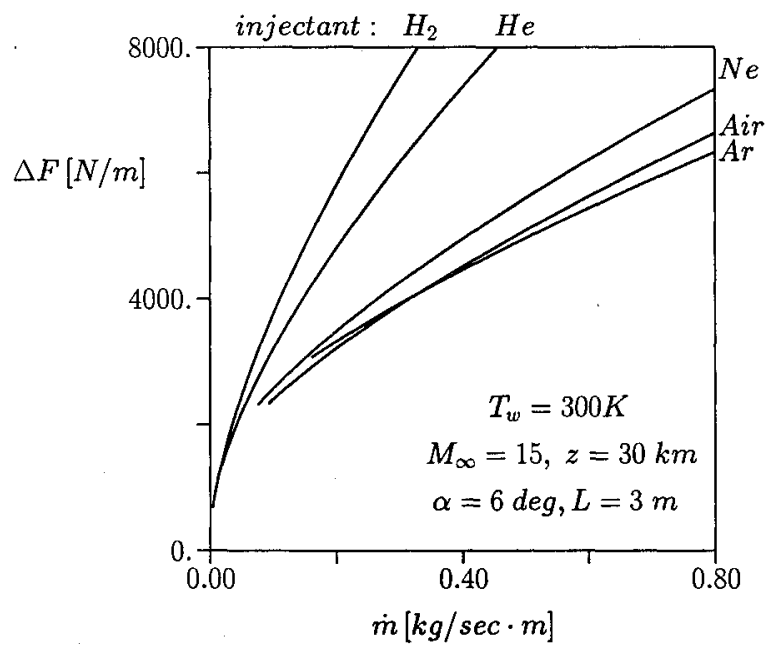

Fig. 6c Force change $\Delta F$ vs mass flow rate $\dot{m}$ for various injectants.
If a simple isothermal model for the atmosphere is adopted and the weak dependence on $\gamma_{b}$ is neglected, then the force change for injection with completely inviscid flow over a wedge with predominantly strong or weak interaction (for $M_{x} \alpha \rightarrow$ $\infty$ ), and constant wall temperature, may be written in the form

$$
\Delta F=\text { const }\left(M_{\infty}^{2} e^{-z / H} L \dot{m}^{2} T_{w} / \sigma_{b}\right)^{1 / 3}
$$

where $H$ is the atmospheric scale height. This simple expression shows the trends indicated in Fig. 6, although not the proper quantitative values. For a fixed mass rate of injected flow, it is seen that $\Delta F$ increases with increasing $M_{\nsim}^{2} e^{-z / H}$, a factor proportional to the freestream dynamic pressure, and also increases as the wedge length $L$ increases; the dependence on $L$ implies that it would be most efficient to distribute a given amount of blown mass over a large surface area. Increasing the ratio of the injectant temperature $T_{w}$ to its molecular mass will cause greater displacement of the outer flow and thereby also increase the force change. The dependence on wedge angle $\alpha$, not present in the above expression, is seen in Fig. $6 \mathrm{~b}$ to be small for the conditions considered there.

\section{Conclusions}

From the results of this analysis, it is concluded that relatively large pressure forces can be achieved with relatively small amounts of gas injected at the surface of a vehicle traveling at hypersonic speeds. Hence, it appears that boundarylayer blowing is a possible alternative to the use of moveable surfaces and/or vectored thrust for control of such a vehicle in the atmosphere, and should be investigated further. Although only the very simple case of two-dimensional wedge flow with a power-law distribution of injected mass is considered in this study, the magnitudes of the surface forces found for relatively small mass flow rates of the blown gas indicate that it is worthwhile to analyze more complex blowing patterns with various injectant gases, in order to obtain desired force distributions, and finally to consider more complex geometries. A numerical code presently under development will be used to accomplish the first of the above extensions to this work.

\section{Acknowledgment}

Support for this research was provided in part by the U.S. Army Strategic Defense Command.

\section{References}

${ }^{1}$ Carter, N. D., and van Leer, B., "Euler Computations of Hypersonic Flow with Strong Blowing,” AIAA Paper 90-0603, Jan. 1990.

${ }^{2}$ Chernyi, G. G., Introduction to Hypersonic Flow, Academic Press, New York, 1961, Chap. 4.

${ }^{3}$ Stewartson, K., The Theory of Laminar Boundary Layers in Com pressible Fluids, Oxford Univ. Press, London, 1964, Chap. 7.

${ }^{4}$ Hayes, W. D., and Probstein, R. F., Hypersonic Flow Theory, Academic Press, New York, 1959, Chap. 9.

${ }^{5}$ Van Dyke, M. D., "A Study of Hypersonic Small-Disturbance Theory," NACA Rept. 1194, 1954 (supersedes TN-3173, 1954).

${ }^{6} \mathrm{Li}$, T.-Y., and Gross, J. F., "Hypersonic Strong Viscous Interaction on a Flat Plate with Surface Mass Transfer," Proceedings of the 1961 Heat Transfer and Fluid Mechanics Institute, pp. 146-160.

${ }^{7}$ Kubota, T., and Fernandez, F. L., "Boundary-Layer Flows with Large Injection and Heat Transfer," AIAA Journal, Vol. 6, No. 1, 1968, pp. 22-28.

${ }^{8}$ Cole, J. D., and Aroesty, J., "The Blowhard Problem-Inviscid Flows with Surface Injection," International Journal of Heat and Mass Transfer, Vol. 11, No. 7, 1968, pp. 1167-1183.

${ }^{9}$ Smith, F. T., and Stewartson, K., "On Slot Injection into a Supersonic Laminar Boundary Layer," Proceedings of the Royal Society of London, Series A, Vol. 332, 1973, pp. 1-22. 
${ }^{10}$ Messiter, A. F., and Matarrese, M. D., "Hypersonic Viscous Interaction with Strong Blowing," Journal of Fluid Mechanics, Vol. 219,1990 , pp. 291-311.

${ }^{11}$ Matarrese, M. D., and Messiter, A. F., "A Numerical Method for the Self-Similar Hypersonic Viscous Shear Layer," Journal of Computational Physics (to be published).

${ }^{12}$ Hirschfelder, J. O., Curtiss, C. F., and Bird, R. B., Molecular Theory of Gases and Liquids, Wiley, New York, 1954, Chap. 8.

${ }^{13}$ Anderson, J. D., Jr., Hypersonic and High-Temperature Gas Dynamics, McGraw-Hill, New York, 1989.
${ }^{14}$ Bird, R. B., Stewart, W. E., and Lightfoot, E. N., Transport Phenomena, Wiley, New York, 1960, Chaps. 1, 8, 16

${ }^{15}$ Hirschfelder, J. O., "Heat Conductivity in Polyatomic or Electronically Excited Gases. II," Journal of Chemical Physics, Vol. 26, No. 2, 1957, pp. 282-285.

${ }^{16}$ Hansen, C. F., "Approximations for the Thermodynamic and Transport Properties of High-Temperature Air," NASA TR R-50, 1959.

${ }^{17}$ JANAF Thermochemical Tables, 2nd ed., U.S. Standard Reference Data System NSRDS-NBS 37, 1971.

\section{Attention Journal Authors: Send Us Your Manuscript Disk}

AIAA now has equipment that can convert virtually any disk $\left(3 \frac{1 / 2-}{2}, 51 / 4-\right.$, or 8 -inch) directly to type, thus avoiding rekeyboarding and subsequent introduction of errors.

The following are examples of easily converted software programs:

- PC or Macintosh $\mathrm{T}^{\mathrm{E} X}$ and $\mathrm{LAT}^{\mathrm{E}} \mathrm{X}$

- PC or Macintosh Microsoft Word

- PC Wordstar Professional

You can help us in the following way. If your manuscript was prepared with a word-processing program, please retain the disk until the review process has been completed and final revisions have been incorporated in your paper. Then send the Associate Editor all of the following:

- Your final version of double-spaced hard copy.

- Original artwork.

- A copy of the revised disk (with software identified).

Retain the original disk.

If your revised paper is accepted for publication, the Associate Editor will send the entire package just described to the AIAA Editorial Department for copy editing and typesetting.
Please note that your paper may be typeset in the traditional manner if problems arise during the conversion. A problem may be caused, for instance, by using a "program within a program"' (e.g., special mathematical enhancements to word-processing programs). That potential problem may be avoided if you specifically identify the enhancement and the word-processing program.

In any case you will, as always, receive galley proofs before publication. They will reflect all copy and style changes made by the Editorial Department.

We will send you an AIAA tie or scarf (your choice) as a "thank you" for cooperating in our disk conversion program. Just send us a note when you return your galley proofs to let us know which you prefer.

If you have any questions or need further information on disk conversion, please telephone Richard Gaskin, AIAA Production Manager, at (202) 646-7496.

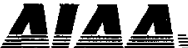

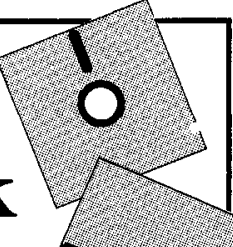
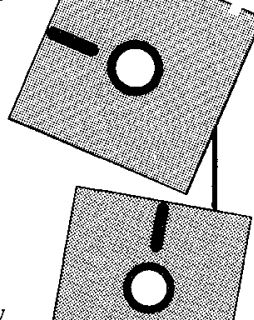
0 